\title{
Leishmania braziliensis: Strain-Specific Modulation of Phagosome Maturation
}

\section{Tamara da Silva Vieira ${ }^{1,2 \dagger}$, Guillermo Arango Duque ${ }^{2 \dagger}$, Kévin Ory ${ }^{2,3}$, Celia Maria Gontijo ${ }^{1}$, Rodrigo Pedro Soares ${ }^{1 *}$ and Albert Descoteaux ${ }^{2 *}$}

${ }^{1}$ Fundação Oswaldo Cruz - FIOCRUZ, Centro de Pesquisas René Rachou, Belo Horizonte, Brazil, ${ }^{2}$ INRS - Centre Armand-Frappier Santé Biotechnologie, Université du Québec, Laval, QC, Canada, ${ }^{3}$ Université de Rennes 1, CHU Rennes, INSERM, Rennes, France

\section{OPEN ACCESS}

Edited by:

Herbert Leonel de Matos Guedes, Federal University of Rio de Janeiro, Brazil

Reviewed by:

Camila I. De Oliveira

Oswaldo Cruz Foundation

(Fiocruz), Brazil

Thiago DeSouza-Vieira,

National Institute of Allergy and Infectious Diseases (NIAID),

United States

Ricardo Silvestre,

University of Minho, Portugal

*Correspondence:

Rodrigo Pedro Soares

rodrigo.pedro@fiocruz.br

Albert Descoteaux

albert.descoteaux@iaf.inrs.ca

tThese authors have contributed equally to this work

Specialty section:

This article was submitted to

Parasite and Host,

a section of the journal

Frontiers in Cellular and Infection

Microbiology

Received: 29 March 2019

Accepted: 26 August 2019

Published: 06 September 2019

Citation:

da Silva Vieira T, Arango Duque G,

Ory K, Gontijo CM, Soares RP and

Descoteaux A (2019) Leishmania

braziliensis: Strain-Specific Modulation

of Phagosome Maturation.

Front. Cell. Infect. Microbiol. 9:319,

doi: 10.3389/fcimb.2019.00319
Leishmania (Viannia) braziliensis is responsible for the largest number of American tegumentary leishmaniasis (ATL) in Brazil. ATL can present several clinical forms including typical (TL) and atypical (AL) cutaneous and mucocutaneous (ML) lesions. To identify parasite and host factors potentially associated with these diverse clinical manifestations, we first surveyed the expression of two virulence-associated glycoconjugates, lipophosphoglycan (LPG) and the metalloprotease GP63 by a panel of promastigotes of Leishmania braziliensis (L. braziliensis) strains isolated from patients with different clinical manifestations of ATL and from the sand fly vector. We observed a diversity of expression patterns for both LPG and GP63, which may be related to strain-specific polymorphisms. Interestingly, we noted that GP63 activity varies from strain to strain, including the ability to cleave host cell molecules. We next evaluated the ability of promastigotes from these L. braziliensis strains to modulate phagolysosome biogenesis in bone marrow-derived macrophages (BMM), by assessing phagosomal recruitment of the lysosome-associated membrane protein 1 (LAMP-1) and intraphagosomal acidification. Whereas, three out of six L. braziliensis strains impaired the phagosomal recruitment of LAMP-1, only the ML strain inhibited phagosome acidification to the same extent as the $L$. donovani strain that was used as a positive control. While decreased phagosomal recruitment of LAMP-1 correlated with higher LPG levels, decreased phagosomal acidification correlated with higher GP63 levels. Finally, we observed that the ability to infect and replicate within host cells did not fully correlate with the inhibition of phagosome maturation. Collectively, our results revealed a diversity of strain-specific phenotypes among L. braziliensis isolates, consistent with the high genetic diversity within Leishmania populations.

Keywords: Leishmania braziliensis, virulence, lipophosphoglycan, GP63, macrophage, phagosome, intracellular survival

\section{INTRODUCTION}

The various species of the protozoan parasite Leishmania cause a spectrum of human diseases ranging from a relatively confined cutaneous lesion to a progressive and potentially fatal visceral infection (Alvar et al., 2012). Upon delivery in the vertebrate host by an infected sand fly, metacyclic Leishmania promastigotes are engulfed by phagocytes. To avoid destruction, these 
parasites have evolved efficient means of disarming the microbicidal functionality of their host cells (Arango Duque and Descoteaux, 2015; Podinovskaia and Descoteaux, 2015; Atayde et al., 2016; Martínez-López et al., 2018). To achieve this, infectious promastigotes rely on a panoply of virulence factors including two abundant components of their surface coat, the glycolipid lipophosphoglycan (LPG) and the GPI-anchored zinc metalloprotease GP63 (Moradin and Descoteaux, 2012; Olivier et al., 2012; Arango Duque and Descoteaux, 2015; Atayde et al., 2016). The use of mutants defective in either LPG or GP63 revealed that these molecules are indeed important for the colonization of phagocytic cells by promastigotes of Leishmania donovani (L. donovani) (Desjardins and Descoteaux, 1997; Lodge et al., 2006), Leishmania major (L. major) (Späth et al., 2000; Joshi et al., 2002), and Leishmania infantum (L. infantum) (LázaroSouza et al., 2018), all of which live in tight individual vacuoles. These virulence factors exert a profound impact on infected cells, altering signaling pathways (Descoteaux et al., 1991; Shio et al., 2012), inducing the production of inflammatory cytokines (Arango Duque et al., 2014), activating the inflammasome (de Carvalho et al., 2019), and inhibiting phagolysosomal biogenesis and functionality (Desjardins and Descoteaux, 1997; Späth et al., 2003; Lodge et al., 2006; Vinet et al., 2009; Matheoud et al., 2013; Matte et al., 2016). Of note, defective synthesis of LPG has no measurable effect on the ability of Leishmania mexicana ( $L$. mexicana), which lives in large communal vacuoles, to replicate in cultured macrophages and cause lesions in mice (Ilg, 2000; Ilg et al., 2001). These findings underline the fact that the relative contribution of a given virulence factor in the ability of promastigotes to colonize mammalian hosts varies among Leishmania species.

Leishmania braziliensis (subgenus Viannia) is responsible for the largest number of American tegumentary leishmaniasis cases in Brazil (ATL) (Alvar et al., 2012; PAHO/WH1O, 2017). ATL may exhibit several clinical forms including typical (TL), atypical (AL), and mucocutaneous (ML) lesions. TL may be confined at the bite site or metastasize to the oronasopharyngeal mucosa to give rise to ML. L. braziliensis AL lesions are scarce and they have been previously reported by Guimarães et al. in Bahia State (Guimarães et al., 2009) and by Quaresma et al. in the Minas Gerais State (Quaresma et al., 2018). Those lesions do not resemble classical TL lesions (round, ulcerated with elevated borders) and their ambiguous nature hinders correct diagnosis. Whether variations in GP63 and LPG levels are associated to the various clinical manifestations of ATL has not been investigated. In this regard, studies aimed at characterizing GP63 in $L$. braziliensis revealed the presence of nearly 40 copies of this gene, as well as important sequence polymorphisms among clinical isolates (Medina et al., 2016). Characterization of LPG from $L$. braziliensis promastigotes revealed structural and compositional similarities to that of $L$. donovani (Soares et al., 2005), as well its strain-dependent capacity to induce inflammatory mediator release (Vieira Td et al., 2019).

To date, studies on the modulation of phagolysosome biogenesis by Leishmania promastigotes and on the contribution of LPG and GP63 to this process have focused mainly on species of the subgenus Leishmania. In the present study, we examined the levels of LPG and GP63 in a panel of L. braziliensis strains and surveyed their ability to interfere with phagosome maturation.

\section{MATERIALS AND METHODS}

\section{Ethics Statement}

This study was carried out in accordance with the recommendations the Canadian Council on Animal Care on animal handling practices. Protocol 1706-07 was approved by the Comité Institutionel de Protection des Animaux of the INRSInstitut Armand-Frappier. Leishmania braziliensis field strains were obtained from patients living in the Xakriabá indigenous community located in São João das Missões municipality, Minas Gerais State, Brazil. Isolates from other endemic areas were obtained from the outpatient care facility at Centro de Referência em Leishmanioses-Instituto René Rachou/Fiocruz Minas from 1993 to 1998. Patient samples were obtained under informed consent procedures approved by the IRR Research Ethics Committee in Human Research, the National Committee for Research Ethics (Comissão Nacional de Ética em PesquisaCONEP) $\mathrm{n}^{\circ} 355 / 2008$, and the National Indian Foundation (Fundação Nacional do Índio-FUNAI) n 149/CGEP/08.

\section{Cell Culture}

Bone marrow-derived macrophages (BMM) were obtained from the bone marrow of 6-8 week-old female C57BL/6 mice and differentiated in complete DMEM [containing L-glutamine (Life Technologies), 10\% v/v heat-inactivated fetal bovine serum (FBS) (Life Technologies), $10 \mathrm{mM}$ HEPES (Bioshop) at pH 7.4, and penicillin-streptomycin (Life Technologies)] supplemented with $15 \% \mathrm{v} / \mathrm{v}$ L929 cell-conditioned medium (LCM) as a source of macrophage colony-stimulating factor. To render BMM quiescent prior to experiments, cells were transferred to tissue culture-treated plates containing glass coverslips for $16 \mathrm{~h}$ in complete DMEM without LCM (Descoteaux and Matlashewski, 1989). BMM were kept in a humidified $37^{\circ} \mathrm{C}$ incubator with $5 \% \mathrm{CO}_{2}$.

Promastigotes were grown in Leishmania medium [M199$1 \mathrm{X}$ (Sigma) with $10 \%$ heat-inactivated FBS, $40 \mathrm{mM}$ HEPES at $\mathrm{pH} 7.4,100 \mu \mathrm{M}$ hypoxanthine, $5 \mu \mathrm{M}$ hemin, $3 \mu \mathrm{M}$ biopterin,

TABLE 1 | Leishmania strains used in this study.

\begin{tabular}{|c|c|c|}
\hline Strain/Isolate & Geographical origin & Lesion type \\
\hline MHOM/BR/75/M2903 & Pará state, Brazil & TL \\
\hline MHOM/BR/1995/RR051 & $\begin{array}{l}\text { Belo Horizonte, Minas } \\
\text { Gerais State }\end{array}$ & $\mathrm{TL}$ \\
\hline MHOM/BR/2009/RR418 & $\begin{array}{l}\text { São João das Missões, } \\
\text { Minas Gerais State }\end{array}$ & $\mathrm{TL}$ \\
\hline MHOM/BR/2008/RR410 & $\begin{array}{l}\text { São João das Missões, } \\
\text { Minas Gerais State }\end{array}$ & $\mathrm{AL}$ \\
\hline MHOM/BR/1996/M15991 & Belém, Pará State & $M L$ \\
\hline IWELL/BR/1981/M8401 & Belém, Pará State & $\begin{array}{l}\mathrm{N} / \mathrm{A} \text { (strain isolated } \\
\text { from vector) }\end{array}$ \\
\hline MHOM/ET/67/Hu3:LV9 & Ethiopia & Visceral \\
\hline $\begin{array}{l}\text { MHOM/SN/74 NIH clone A2 } \\
\text { (A2WF) }\end{array}$ & Senegal & $\mathrm{TL}$ \\
\hline
\end{tabular}


$1 \mu \mathrm{M}$ biotin, MEM vitamin solution $1 \mathrm{X}$, and penicillinstreptomycin] in a $26^{\circ} \mathrm{C}$ incubator (Soares et al., 2002; Arango Duque et al., 2014). The L. braziliensis strains used in this study (Table 1) include the World Health Organization reference strain (MHOM/BR/75/M2903), a ML isolate (MHOM/BR/1996/M15991), and a strain derived from $P$. welcomei sand flies (IWELL/BR/1981/M8401). The L. braziliensis RR051 strain was isolated from a TL lesion in the Minas Gerais State, and the RR418 (TL) and RR410 (AL) strains from lesions found in the Xakriabá community (Quaresma et al., 2018). These strains have been molecularly typed as L. braziliensis (Quaresma et al., 2018; Rugani et al., 2018). Leishmania donovani LV9 and L. major Seidman A2 promastigotes were freshly differentiated from splenic or ear lesion-derived amastigotes, respectively.

\section{Infections and Phagosome Acidification Assays}

Late stationary phase promastigotes (5-day cultures at $>50$ $\times 10^{6}$ promastigotes $/ \mathrm{ml}$ ) from an early passage, or zymosan particles, were opsonised with serum from C5-deficient DBA/2 mice, resuspended in cold complete DMEM and fed to BMM (10:1 ratio) that had been seeded onto glass coverslips. Cells were incubated at $4^{\circ} \mathrm{C}$ for $5 \mathrm{~min}$, and centrifuged for $2 \mathrm{~min}$ at 1,200 rpm (Arango Duque et al., 2013). Particle internalization was triggered by transferring cells to $37^{\circ} \mathrm{C}$ (Vinet et al., 2008; Arango Duque et al., 2014). Two hours post-internalization, infected macrophages were washed $3 \mathrm{X}$ with $1 \mathrm{ml}$ warm DMEM to remove non-internalized promastigotes. Macrophages were either left at $37^{\circ} \mathrm{C}$ for an extra $22 \mathrm{~h}$, or prepared for confocal microscopy. To assay phagosome acidification, BMM were incubated for $2 \mathrm{~h}$ with the acidotropic LysoTracker Red dye (diluted 1:1,000; Molecular probes) prior to the $2 \mathrm{~h}$ infection. In the case of the $24 \mathrm{~h}$ infection, infected macrophages were incubated in diluted LysoTracker for $2 \mathrm{~h}$ prior to the end of the infection time point. Cells were then washed and fixed.

For intracellular colonization assays, 6, 24, and $72 \mathrm{~h}$-infected BMM seeded on coverlips were washed with PBS1X, stained with the Hema $3^{\text {TM }}$ Stat Pack (Fisher), briefly washed with deionized water, and air-dried for $10 \mathrm{~min}$. Coverslips were mounted onto a drop of Fluoromount-G and sealed. Images were acquired with a Qimaging camera (Teledyne Technologies International Corp) mounted on a Nikon Eclipse E800 microscope (60X objective). Images were compiled and analyzed with the ImageJ (Rueden et al., 2017) interphase of the Icy image analysis software (de Chaumont et al., 2012). Threshold segmentation was used to differentiate and enumerate BMM and intracellular Leishmania nuclei.

\section{Confocal Immunofluorescence Microscopy}

Infected cells on coverslips were fixed with $2 \%$ paraformaldehyde (Thermo Scientific) for $20 \mathrm{~min}$ and blocked and permeabilized for 17 min with a solution of $0.1 \%$ Triton X-100, $1 \%$ BSA, $6 \%$ non-fat milk, $20 \%$ goat serum, and 50\% FBS. This was followed by a $2 \mathrm{~h}$ incubation with a monoclonal rat antibody to Lysosomeassociated membrane protein 1 (LAMP-1) (developed by J. T. August (1D4B) and purchased through the Developmental Studies Hybridoma Bank at the University of Iowa and the
National Institute of Child Health and Human Development) diluted 1:200 in PBS1X. Subsequently, cells were incubated for $35 \mathrm{~min}$ in a solution containing an anti-rat antibody conjugated to Alexa-488 (diluted 1:500; Molecular Probes) and DAPI (1:40,000; Molecular Probes). Coverslips were washed three times with PBS1X after every step. After the final wash, coverslips were mounted cell-side facing a drop of Fluoromount-G (Southern Biotechnology Associates) that was placed on a glass slide (Fisher); coverslips were sealed with nail polish (Sally Hansen). Infected macrophages were imaged with the $63 \mathrm{X}$ objective of an LSM780 confocal microscope (Carl Zeiss Microimaging) and image processing was done with the ZEN 2012 software. In regards to LysoTracker-treated cells, fixed samples were incubated in diluted DAPI for $35 \mathrm{~min}$ prior to mounting. Recruitment was evaluated by scoring the presence of staining on the phagosome membrane (LAMP-1) and/or the phagosome lumen (LysoTracker) (Vinet et al., 2009; Arango Duque et al., 2014). One hundred phagosomes per coverslip were scored for every experimental condition, each done in duplicate.

\section{Electrophoresis, Western Blotting, and Zymography}

Before lysis, stationary phase promastigotes, or infected BMM were washed with cold PBS1X containing $1 \mathrm{mM}$ sodium orthovanadate and $5 \mathrm{mM} \mathrm{1,10-phenanthroline} \mathrm{(Sigma).} \mathrm{Pelleted}$ parasites were resuspended in a lysis buffer containing $1 \%$ NP-40, $50 \mathrm{mM}$ Tris- $\mathrm{HCl}$ (pH 7.5), $150 \mathrm{mM} \mathrm{NaCl}, 1 \mathrm{mM}$ EDTA (pH 8), $15 \mathrm{mM}$ 1,10-phenathroline and phosphatase and protease inhibitors (Roche). Samples were incubated at $-70^{\circ} \mathrm{C}$, sonicated with $20 \mathrm{~s}$ and thereafter centrifuged at $4^{\circ} \mathrm{C}$ for $15 \mathrm{~min}$ to remove insoluble material. After protein quantification, $30 \mu \mathrm{g}$ of protein $(10 \mu \mathrm{g}$ in the case of promastigote lysate) was boiled $\left(100^{\circ} \mathrm{C}\right)$ for $6 \mathrm{~min}$ in SDS sample buffer and migrated in SDS-PAGE gels. Proteins were transferred onto PDVF (phosphoglycan blots) or nitrocellulose membranes and thereafter blocked for $2 \mathrm{~h}$ in TBS1X-0.1\% Tween containing 5\% BSA. Membranes were subsequently probed (overnight at $4^{\circ} \mathrm{C}$ ) with a mouse mAb (CA7AE; Cedarlane) that recognizes unbranched $\mathrm{Gal} \beta 1,4 \mathrm{Man} \alpha 1-\mathrm{PO}_{4}$ phosphoglycan moieties (Tolson et al., 1989), and detects L. braziliensis LPG (Vieira Td et al., 2019). Membranes were also probed for the presence of GP63 (mouse mAb \#235 targeting the membraneanchored version of L. major GP63) (Button et al., 1993; Macdonald et al., 1995); VAMP8 and syntaxin-5 (Stx5) (rabbit pAbs from Synaptic Systems). Anti-L. donovani aldolase (rabbit $\mathrm{pAb}$, a kind gift from A. Jardim) and $\beta$-actin (Sigma) were used as loading controls. After washing, membranes were probed with suitable HRP-conjugated secondary antibodies for $1 \mathrm{~h}$ at room temperature, and incubated in ECL (GE Healthcare). Immunodetection was achieved via chemiluminescence (Arango Duque et al., 2014). To assess GP63 proteolysis via zymography, lysates were incubated at $50^{\circ} \mathrm{C}$ for $5 \mathrm{~min}$ in sample buffer without DTT, and then migrated in 10-12\% SDS-PAGE gels containing $0.12 \%$ gelatin (Sigma) (Hassani et al., 2014; Arango Duque et al., 2019). Gels were incubated for $2 \mathrm{~h}$ in the presence of $50 \mathrm{mM}$ Tris $\mathrm{pH} 7.4,2.5 \%$ Triton $\mathrm{X}-100,5 \mathrm{mM} \mathrm{CaCl}_{2}$ and 


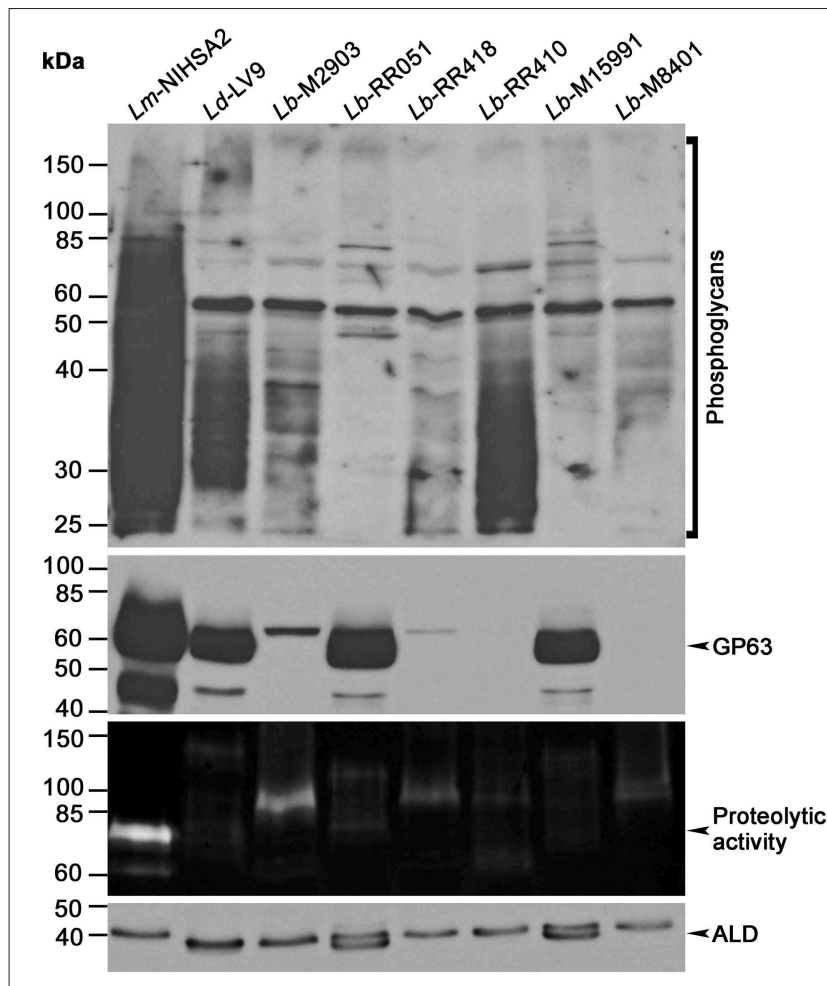

FIGURE 1 | Expression of virulence-associated glycoconjugates in different $L$. braziliensis strains. Leishmania promastigote lysates were assayed for LPG, GP63, and aldolase expression by Western blot, and GP63 activity was probed via gelatin zymography. Images are representative of two independent experiments. $L m$, L. major; $L d, L$. donovani; $L b, L$. braziliensis; $A L D, L d$ aldolase.

$1 \mathrm{mM} \mathrm{ZnCl}$, followed by an overnight incubation at $37^{\circ} \mathrm{C}$ in a buffer containing $50 \mathrm{mM}$ Tris $\mathrm{pH} 7.4,5 \mathrm{mM} \mathrm{CaCl}_{2}, 1 \mathrm{mM} \mathrm{ZnCl}_{2}$, and $0.01 \% \mathrm{NaN}_{3}$. Protease activity was visualized by gradually distaining gels that were incubated in $0.5 \%$ Coomassie Brilliant blue (Sigma) for $1 \mathrm{~h}$.

\section{Statistical Analysis}

Statistical differences in recruitment levels were assessed using one-way ANOVA followed by Bonferroni post-hoc tests. Data were considered statistically significant when $p<0.05$ and univariate column scatter graphs were constructed using GraphPad Prism 6.0 (GraphPad Software Inc).

\section{RESULTS}

\section{Expression of LPG and GP63 Varies Among Strains of $L$. braziliensis}

The ability of Leishmania promastigotes to colonize host cells and impair phagosome maturation and functionality is mediated to a large extent by the virulence factors LPG and GP63 (Chaudhuri et al., 1989; Späth et al., 2003; Moradin and Descoteaux, 2012; Atayde et al., 2016; Matte and Descoteaux, 2016). Here, we sought to determine the relative levels of LPG and GP63 expressed by promastigotes of a panel of L. braziliensis strains differing in their origin (Table 1). We included in our analysis $L$. major (NIH Seidman A2) and L. donovani (LV9) promastigotes as controls. Western blot analysis performed on promastigote lysates showed notable variations in the levels of LPG among the tested strains (Figure 1). Particularly, whereas the levels of LPG expressed by L. braziliensis RR410 were similar to those observed for $L$. donovani LV9, the levels detected in the other $L$. braziliensis strains were lower. In the case of GP63, we observed important differences among the L. braziliensis strains (Figure 1). Both L. braziliensis strains RR051 and M15991 expressed GP63 at levels comparable to those observed for L. donovani LV9. In contrast, GP63 levels were very low in the other strains. Interestingly, when we assessed the proteolytic activity of GP63 present in the Leishmania promastigotes lysates, we observed a lack of correlation with the GP63 levels detected by Western blot (Figure 1). Notably, L. braziliensis strains with low levels of GP63 (M2903 and RR418) showed high GP63 proteolytic activity, whereas L. braziliensis strains expressing higher GP63 levels (RR051 and M15991) showed reduced GP63 activity. These observations clearly demonstrated important intra-specific variations in the levels of detected LPG and GP63 (as well as GP63 activity) expressed by $L$. braziliensis strains isolated from patient with diverse ATL manifestations and from the insect vector.

\section{Cleavage of GP63 Substrates by L. braziliensis Strains}

Given the variations in GP63 levels and activity observed among the L. braziliensis isolates, we investigated the impact of these differences on the cleavage of phagosomal host cell proteins known to be targeted by GP63 (Matheoud et al., 2013). To this end, we performed Western blot analyses to assess the levels and integrity of the soluble $N$-ethylmaleimide-sensitive-factor attachment protein receptors (SNAREs) VAMP8 and Stx5, in lysates of BMM infected for $6 \mathrm{~h}$ with promastigotes of selected L. braziliensis strains (M2903, RR418, M15991, and M8401) and promastigotes of L. major NIHS A2 as control. As shown in Figure 2, VAMP8 was cleaved to the same extent by all $L$. braziliensis strains and by L. major NIHS A2, regardless of the levels and activity of GP63 detected in the cell lysates. In contrast, cleavage of the endoplasmic reticulum (ER)- and Golgi-resident SNARE Stx5 was strain-dependent and did not entirely correlate with the levels and activity of GP63 detected in the cell lysates (Figure 2). Collectively, these results indicate that cleavage of host cell GP63 substrates occurs in BMM infected with all $L$. braziliensis strains tested, albeit with some differences in the extent of cleavage. These findings also suggest that sensitivity to GP63 cleavage is substrate-specific.

\section{L. braziliensis Impairs Phagosomal Recruitment of LAMP-1 in a Strain-Specific Manner}

Given the variations observed among our panel of L. braziliensis strains in LPG and GP63 levels and activity, as well as substrate cleavage, we investigated the impact of $L$. braziliensis promastigotes on phagosome maturation. To this end, we incubated BMM with promastigotes from our panel of L. braziliensis strains for 2 and $24 \mathrm{~h}$ and assessed the 


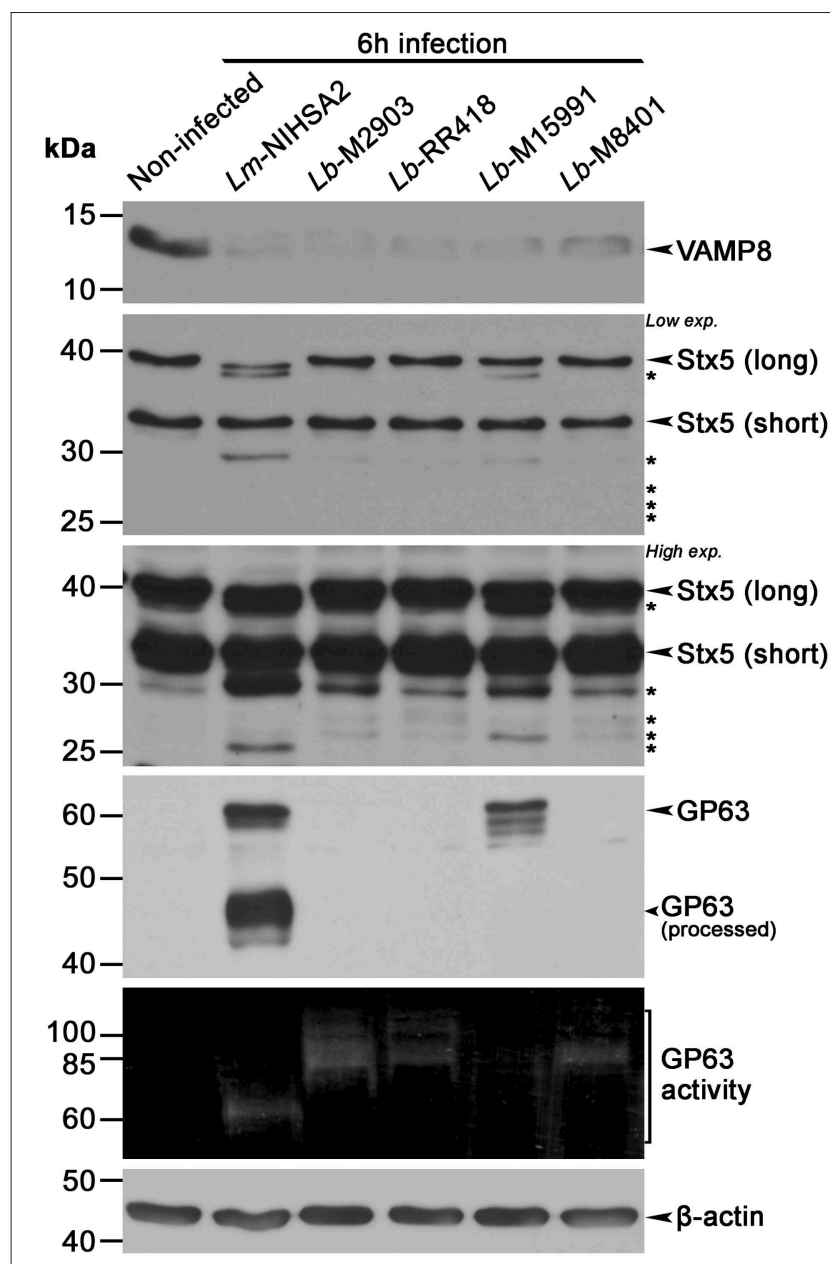

FIGURE 2 | Proteolytic cleavage of phagosome-associated proteins by Leishmania braziliensis (L. braziliensis). BMM were infected with opsonized stationary phase promastigotes from selected $L$. braziliensis strains, and the cleavage of phagosomal proteins VAMP8 and Stx5 was assessed via Western blot. GP63 activity was also assayed via gelatin zymography and $\beta$-actin was used as loading control. The long form of Stx5 is localized at the Golgi and ER, and the short one at the Golgi. Asterisks denote cleavage fragments. $L m, L$. major; Lb, L. braziliensis.

recruitment of the lysosomal marker LAMP-1 to phagosomes. Promastigotes of L. donovani (LV9 strain), which efficiently inhibit phagosome maturation and phagosomal recruitment of LAMP-1 (Scianimanico et al., 1999), and zymosan were used as controls. At $2 \mathrm{~h}$ after the initiation of phagocytosis, we observed a higher recruitment of LAMP-1 to phagosomes containing L. braziliensis strains RR051 and M15991 compared to phagosomes containing L. donovani LV9 (Figures 3A,B). As expected, recruitment of LAMP-1 to phagosomes containing zymosan was higher to that observed for phagosomes induced by promastigotes of $L$. donovani LV9 and of those from $L$. braziliensis isolated from an AL lesion (RR410) (Figure 3). At $24 \mathrm{~h}$ post-infection, the presence of LAMP-1 on phagosomes harboring $L$. donovani promastigotes LV9 remained very low, as was also the case for phagosomes containing L. braziliensis
M2903, RR410, and RR418. However, recruitment of LAMP1 to phagosomes harboring L. braziliensis RR051 and M8401 was significantly higher than the levels observed for phagosomes containing the other L. braziliensis strains and L. donovani LV9 (Figure 3). These results suggest that the ability L. braziliensis promastigotes to interfere with phagosome maturation varies among strains.

\section{Phagosome Acidification Is Differentially Modulated by $L$. braziliensis Strains}

Consistent with their ability to inhibit phagolysosome biogenesis (Desjardins and Descoteaux, 1997), we previously reported that $L$. donovani promastigotes efficiently impair phagosome acidification (Vinet et al., 2009). To further characterize the impact of L. braziliensis promastigotes on phagosome maturation, we used the lysotropic dye LysoTracker Red to monitor acidification kinetics of phagosomes harboring the various L. braziliensis strains. Consistent with previous studies, at $2 \mathrm{~h}$ post-infection, acidification occurred in the majority of zymosan-harboring phagosomes but was hindered in phagosomes containing L. donovani LV9 promastigotes (Figures 4A,B). Similar impairment of phagosome acidification was observed for all L. braziliensis strains, with the exception of the strain isolated from an AL lesion (RR410) (Figures 4A,B). At $24 \mathrm{~h}$ post-infection, most phagosomes harboring $L$. donovani LV9 (80\%) and the L. braziliensis ML isolate (M15991) (70\%) remained negative for LysoTracker Red (Figure 4). In contrast, over $70 \%$ of phagosomes containing L. braziliensis isolates RR418 and RR410 were positive for LysoTracker Red at $24 \mathrm{~h}$ (Figure 4B). These data indicate that most L. braziliensis strains in our panel inhibit phagosome acidification during the early phase of macrophage infection. However, at later time points, the capacity to hinder phagosome acidification varies in a strainspecific manner.

\section{Colonization of Macrophages by L. braziliensis Strains Does Not Fully Correlate With the Ability to Inhibit Phagosome Maturation and Acidification}

Previous studies with $L$. donovani and L. major (Desjardins and Descoteaux, 1997; Späth et al., 2003; Vinet et al., 2009) revealed a correlation between the ability of these parasites to impair phagosome maturation and the ability to colonize macrophages. To investigate whether such a correlation exists for the L. braziliensis strains under study, we incubated BMM for $2 \mathrm{~h}$ with promastigotes of selected strains (M2903, RR418, M15991, and M8401) and promastigotes of L. major NIHS A2 as control. We then quantified the number of parasites per 100 macrophages and the percentage of infected macrophages at 6 , 24, and $72 \mathrm{~h}$ post-phagocytosis. As shown in Figure 5, the ability to survive and replicate over time within BMM varied among the L. braziliensis strains analyzed. At the exception of strain M2903, which displayed reduced ability to survive in BMM over $72 \mathrm{~h}$, all the other strains persisted and two of them (RR418 and M8401) replicated as was the case for L. major NIHS A2. Interestingly, L. braziliensis strain M2903, which survived poorly in BMM, 

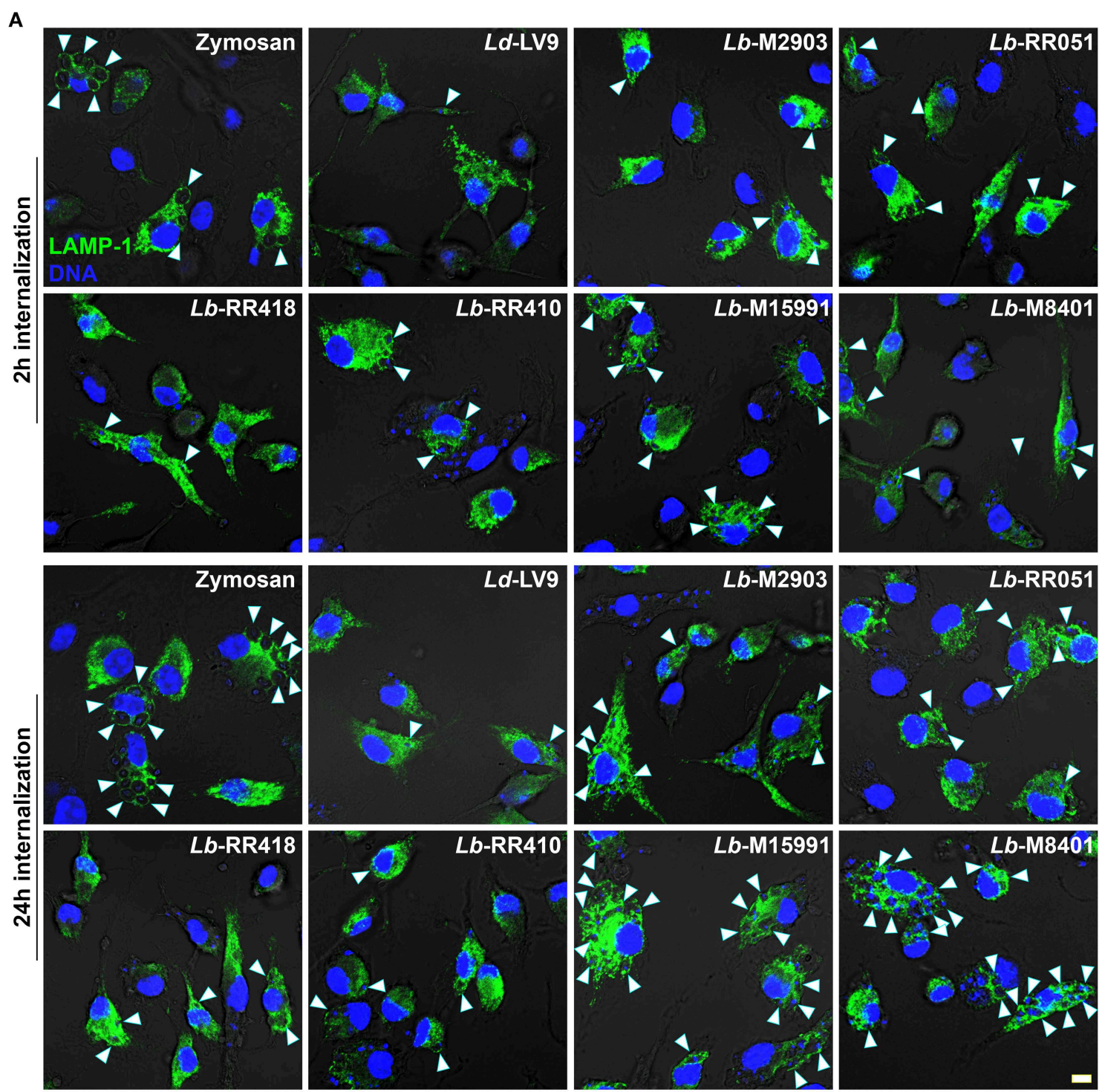

B
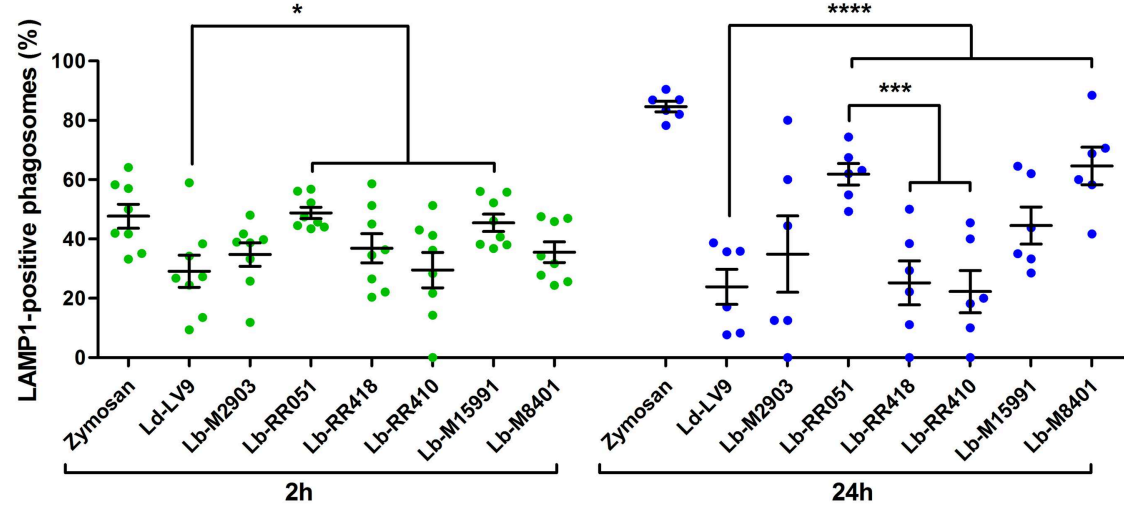

FIGURE 3 | LAMP-1 recruitment to parasitophorous vacuoles harboring L. braziliensis parasites. (A) BMM were infected with opsonized stationary phase promastigotes from different L. braziliensis strains, and LAMP-1 (green) recruitment to parasite-containing phagosomes was visualized via immunofluorescence at 2 and $24 \mathrm{~h}$ post-internalization. DNA is shown in blue; scale bar $=5 \mu \mathrm{m}$. White arrowheads indicate LAMP1 recruitment. (B) Quantification of LAMP-1-positive phagosomes at $2 \mathrm{~h}$ (green dots) and $24 \mathrm{~h}$ (blue dots) post-phagocytosis. Bars depict the mean \pm SEM of \% positive phagosomes counted over three independent experiments done at least in duplicate coverslips. ${ }^{*} p<0.05 ;{ }^{* \star *} p<0.001 ;{ }^{* \star \star *} p<0.0001$. Ld, L. donovani; Lb, L. braziliensis. 

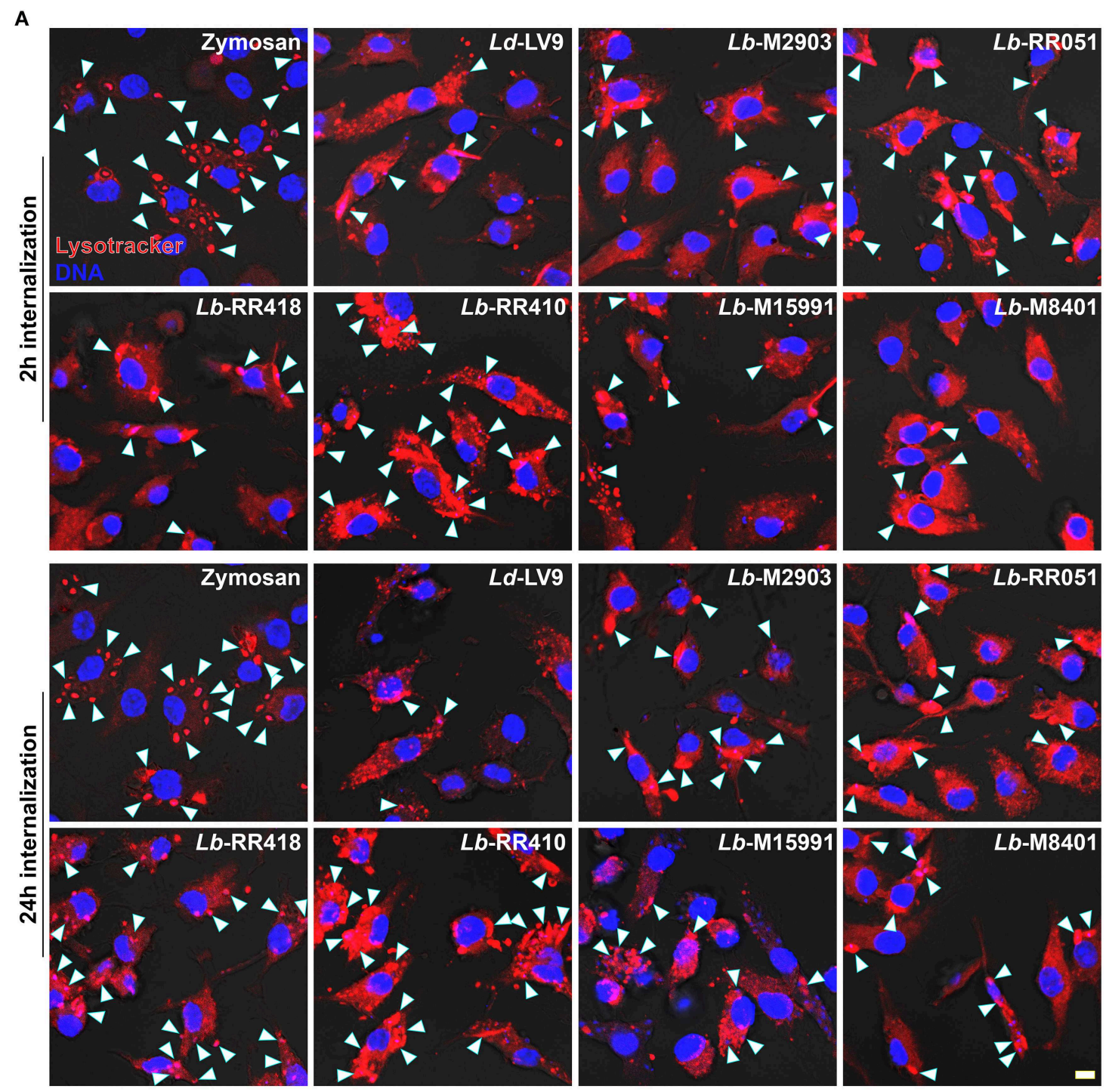

B

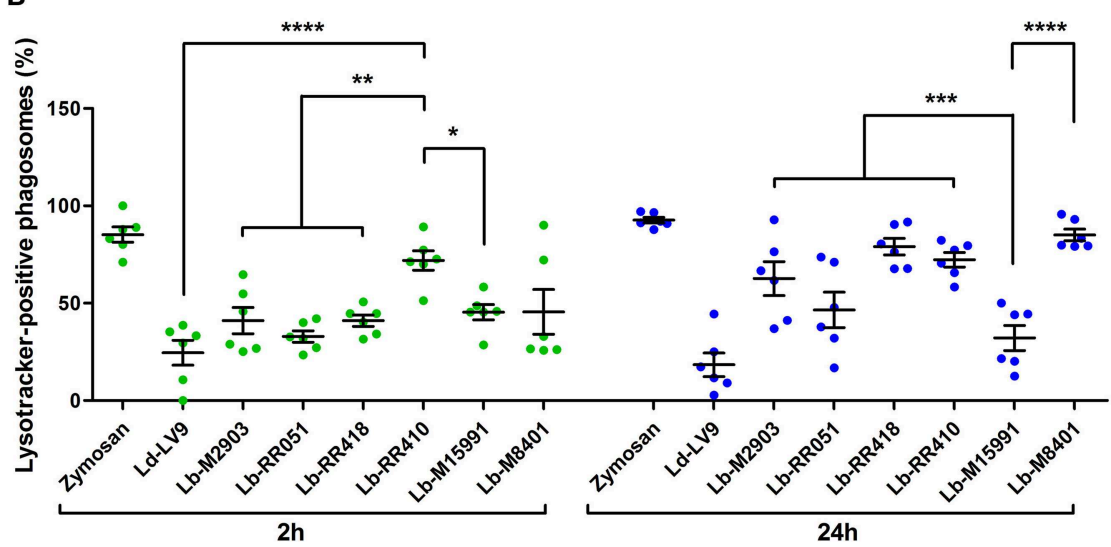

FIGURE 4 | Acidification of parasitophorous vacuoles harboring L. braziliensis parasites. (A) Bone marrow-derived macrophages (BMM) were infected with opsonized stationary phase promastigotes from different $L$. braziliensis strains, and acidification of parasite-containing vacuoles was assayed via LysoTracker staining (red) at 2 and $24 \mathrm{~h}$ post-internalization. DNA is shown in blue; scale bar $=5 \mu \mathrm{m}$. White arrowheads indicate LysoTracker-positive phagosomes. (B) Quantification of LysoTracker-positive phagosomes at $2 \mathrm{~h}$ (white bars) and $24 \mathrm{~h}$ (gray bars) post-phagocytosis. Bars depict the mean \pm SEM of \% positive phagosomes counted over three independent experiments done in duplicate. ${ }^{\star} p<0.05 ;{ }^{* \star} p<0.01 ;{ }^{* \star \star} p<0.001 ;{ }^{\star \star \star *} p<0.0001$. Ld, L. donovani; Lb, L. braziliensis. 




B

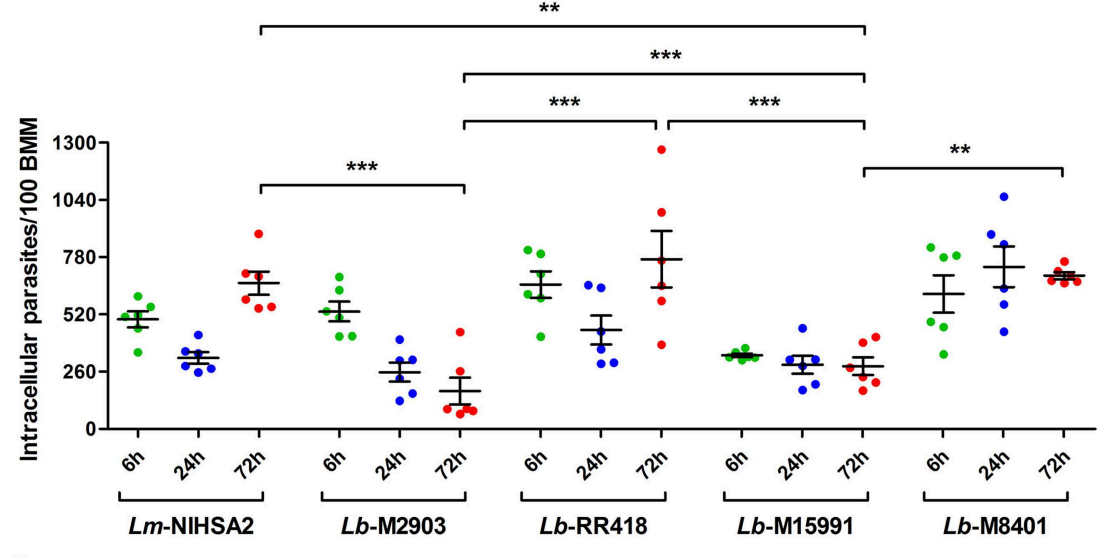

C
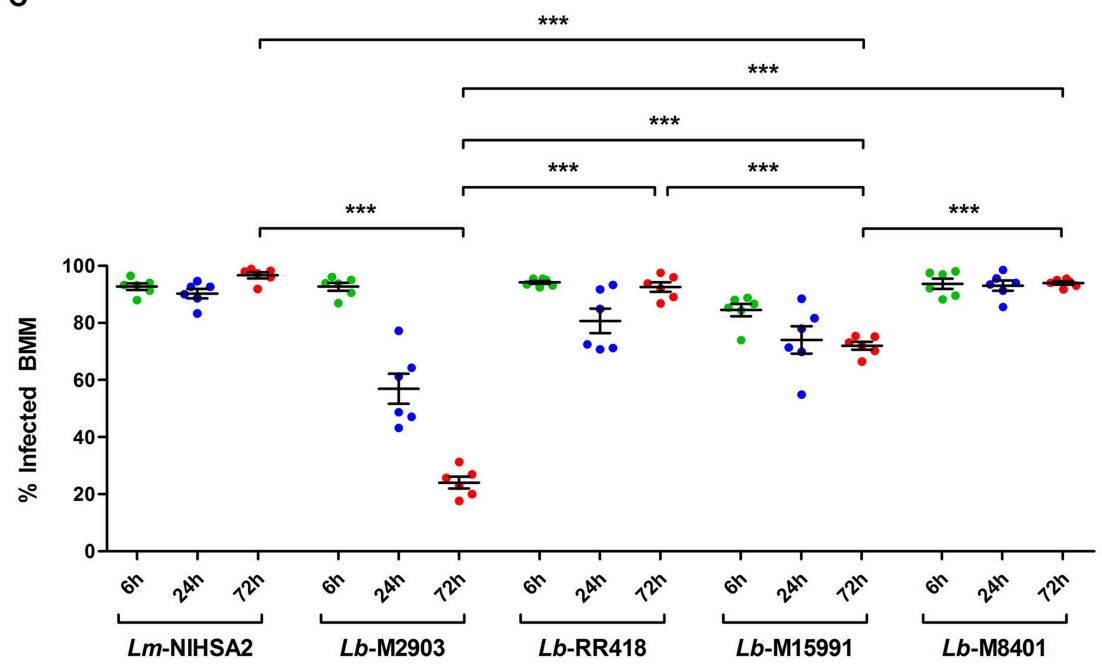

FIGURE 5 | Intracellular survival and replication of $L$. braziliensis parasites. BMM were infected with opsonized stationary phase promastigotes from selected $L$. braziliensis strains, and intracellular survival was quantified in Hema 3-stained cells. (A) Representative images of the $72 \mathrm{~h}$ time point. Scale bar $=10 \mu \mathrm{m}$. Intracellular survival at 6 (green dots), 24 (blue dots), and $72 \mathrm{~h}$ (red dots) post-infection was assessed via the quantification of internalized parasites in 100 macrophages (B) and the percentage of infected cells $\mathbf{( C )}$. In both (B,C), bars depict the mean \pm SEM of two independent experiments done in triplicate coverslips. ${ }^{* \star} p<0.01$;

${ }^{\star \star \star} p<0.001$. Lm, L. major; Lb, L. braziliensis. 
was among the most efficient strains at inhibiting phagosome maturation (Figures 3, 4). For strains RR418 and M8401, their ability to replicate in BMM correlated with their capacity to impair the phagosomal recruitment of LAMP-1 and acidification during the early phases of infection (Figures 3, 4). These data are consistent with the notion that factor(s) other than the capacity to impair phagosome maturation are required for colonization of host cells by L. braziliensis.

\section{DISCUSSION}

The Leishmania virulence factors LPG and GP63 contribute to the ability of promastigotes to colonize phagocytic cells by targeting key host cell host defense mechanisms, including the biogenesis of microbicidal phagolysosomes. In the present study, we sought to examine the levels of LPG and GP63 expressed by promastigotes of $L$. braziliensis (subgenus Viannia) strains isolated from patients exhibiting various clinical manifestations of ATL and from the insect vector. We also characterized the ability of these L. braziliensis strains to impair phagosome maturation and to infect and replicate within macrophages.

Our results revealed an unexpected diversity of expression patterns for both LPG and GP63 among the evaluated $L$. braziliensis strains. Although some strains expressed LPG levels similar to those of $L$. donovani LV9 promastigotes, other strains expressed very low LPG levels. Similarly, some L. braziliensis strains expressed GP63 levels comparable to those observed in L. donovani LV9, whereas other strains expressed very low GP63 levels. Interestingly, we noted that GP63 activity varies from strain to strain, and does not correlate with GP63 levels detected by Western blot. Whether the polymorphims detected in the GP63 genes of L. braziliensis (Medina et al., 2016) affected the recognition of GP63 by our anti-GP63 antibody is however unclear. Clearly, the significance of these observations deserves to be further investigated.

As part of their strategy to colonize host phagocytes, Leishmania promastigotes alter the composition and properties of the parasitophorous vacuole (Moradin and Descoteaux, 2012; Séguin and Descoteaux, 2016). Phagosomal recruitment of the lysosomal protein LAMP-1 is a widely used marker of phagosome maturation (Huynh et al., 2007). In the case of Leishmania promastigotes, delayed phagosomal acquisition of LAMP-1 following phagocytosis supported the notion that these parasites impair phagolysosomal biogenesis (Scianimanico et al., 1999; Lerm et al., 2006; Verma et al., 2017). Interestingly, we found that the ability to inhibit the phagosomal recruitment of LAMP-1 varies significantly among our panel of $L$. braziliensis strains. Phagosome acidification is an important consequence of the maturation process and we previously reported that it is efficiently inhibited by $L$. donovani promastigotes (Vinet et al., 2009). Similar to the recruitment of LAMP-1, we observed an important variation among promastigotes of the $L$. braziliensis strains tested in their capacity to inhibit phagosomal acidification. Interestingly, whereas promastigotes of $L$. donovani LV9 efficiently inhibited both phagosome acidification and recruitment of LAMP-1, we observed no correlation between the ability to inhibit phagosomal recruitment of LAMP-1 and phagosome acidification among the L. braziliensis strains. Previous work from our group revealed that acquisition of LAMP-1 and of the v-ATPase by phagosomes occurs through two distinct mechanisms (Vinet et al., 2009). In the case of L. donovani, LPG is the molecule responsible for inhibiting both the phagosomal recruitment of LAMP-1 and acidification (Scianimanico et al., 1999; Vinet et al., 2009). However, the ability of $L$. braziliensis strains to interfere with phagosome maturation does not appear to correlate with LPG levels.

In addition to LPG, Leishmania promastigotes use the metalloprotease GP63 to modulate the composition and function of phagosomes through the cleavage of host proteins such as VAMP3, VAMP8, and Synaptotagmin XI (Matheoud et al., 2013; Arango Duque et al., 2014; Casgrain et al., 2016; Matte and Descoteaux, 2016; Matte et al., 2016). Since VAMP8 is required for antigen cross-presentation (Matheoud et al., 2013), its cleavage by the various L. braziliensis strains suggests that they efficiently inhibit antigen cross-presentation. Future experiments will specifically address this issue. On the other hand, the endoplasmic reticulum- and Golgi-resident SNARE Stx5 is partially cleaved, to varying extents, by our L. braziliensis strains. This SNARE regulates trafficking between the phagosome and the secretory pathway (Cebrian et al., 2011; Arango Duque et al., 2019) and contributes to the expansion of communal parasitophorous vacuoles harboring L. amazonensis (Canton and Kima, 2012). The significance of its cleavage by L. braziliensis for establishment and replication within macrophages is an issue that will deserve further investigation.

In L. braziliensis, GP63 is present on chromosome 10 and strains isolated from different clinical manifestations from the same geographical region have conserved domains and display specific polymorphisms in their catalytic sites (Medina et al., 2016; Sutter et al., 2017; Quaresma et al., 2018). This variability could result in different virulence patterns and clinical outcomes. Of interest, a recent genomic analysis of Leishmania clinical isolates revealed important differences among genetically highly related Leishmania strains, including both in amplification and in loss of genes linked to parasite infectivity such as GP63 (Bussotti et al., 2018). Whether the diversity of GP63 levels and activity portrayed by the L. braziliensis strains is the consequence of gene amplification associated to environmental adaptation is a likely possibility that deserves further investigation. Similar to LPG and GP63, GIPLs are highly expressed on the Leishmania surface (Assis et al., 2012). They are inhibitory molecules impairing $\mathrm{NO}$ and cytokine production by murine macrophages and their role on phagosome maturation and intracellular survival will be assayed in prospective studies. Together, our findings underline the importance of performing functional genetic analyses with these clinical L. braziliensis strains to directly assess the importance of LPG and GP63 in the colonization of host phagocytes, and ultimately in the pathogenesis of ATL.

For the past several decades, research on virulence or immune subversion mechanisms of Leishmania has been for the most part performed with reference or laboratory strains. Results obtained with those strains allowed for the discovery of several biological processes. For instance, the Th1/Th2 dichotomy and 
the importance of IL-4 in mediating susceptibility to infection were discovered using a particular L. major strain (Heinzel et al., 1989). However, studies using other L. major strains led to opposite results (Noben-Trauth et al., 1996, 1999). In the case of $L$. braziliensis ATL strains, our study revealed an unexpected diversity in terms of expression of virulence molecules and ability to interfere with phagosome maturation. Clearly, these studies highlight the fact that it is important to exert caution when drawing broad conclusions based on observations obtained with a single strain or isolate of a given Leishmania species.

\section{DATA AVAILABILITY}

All datasets generated for this study are included in the manuscript and/or the supplementary files.

\section{ETHICS STATEMENT}

Mice were manipulated under the guidelines of protocol 170607 of the Comité Institutionel de Protection des Animaux of the INRS-Institut Armand-Frappier, which respects animal handling practices promulgated by the Canadian Council on Animal Care. Leishmania braziliensis field strains were obtained from patients living in the Xakriabá indigenous community located in São João das Missões municipality, Minas Gerais State, Brazil. Isolates from other endemic areas were obtained from the outpatient care facility at Centro de Referência em Leishmanioses-Instituto René Rachou/Fiocruz Minas from 1993 to 1998. Patient samples were obtained under informed consent procedures approved by the IRR Research Ethics Committee in Human Research, the National Committee for Research Ethics (Comissão Nacional de Ética em Pesquisa-CONEP) n ${ }^{\circ} 355 / 2008$, and the National

\section{REFERENCES}

Alvar, J., Vélez, I. D., Bern, C., Herrero, M., Desjeux, P., Cano, J., et al. (2012). Leishmaniasis worldwide and global estimates of its incidence. PLoS ONE 7:e35671. doi: 10.1371/journal.pone.0035671

Arango Duque, G., and Descoteaux, A. (2015). Leishmania survival in the macrophage: where the ends justify the means. Curr. Opin. Microbiol. 26, 32-40. doi: 10.1016/j.mib.2015.04.007

Arango Duque, G., Fukuda, M., and Descoteaux, A. (2013). Synaptotagmin XI regulates phagocytosis and cytokine secretion in macrophages. J. Immunol. 190, 1737-1745. doi: 10.4049/jimmunol.1202500

Arango Duque, G., Fukuda, M., Turco, S. J., Stäger, S., and Descoteaux, A. (2014). Leishmania promastigotes induce cytokine secretion in macrophages through the degradation of synaptotagmin XI. J. Immunol. 193, 2363-2372. doi: 10.4049/jimmunol.1303043

Arango Duque, G., Jardim, A., Gagnon, É., Fukuda, M., and Descoteaux, A. (2019). The host cell secretory pathway mediates the export of Leishmania virulence factors out of the parasitophorous vacuole. PLoS Pathog. 15:e1007982. doi: 10.1371/journal.ppat.1007982

Assis, R. R., Ibraim, I. C., Noronha, F. S., Turco, S. J., and Soares, R. P. (2012). Glycoinositolphospholipids from Leishmania braziliensis and L. infantum: Modulation of Innate Immune System and Variations in Carbohydrate Structure. PLoS Neglected Tropical Dis. 6:e1543. doi: 10.1371/journal.pntd.0001543

Atayde, V. D., Hassani, K., da Silva Lira Filho, A., Borges, A. R., Adhikari, A., Martel, C., et al. (2016). Leishmania exosomes and other virulence factors:
Indian Foundation (Fundação Nacional dÍo ndio-FUNAI) $\mathrm{n}^{\circ}$ 149/CGEP/08.

\section{AUTHOR CONTRIBUTIONS}

GA, TS, RS, and AD conceived and designed the study, contributed to the data analysis, and drafted and revised the manuscript. GA, TS, and KO performed the experiments. CG provided the L. braziliensis strains. GA, TS, RS, and AD wrote and revised the manuscript. All authors read and approved the final version of this manuscript.

\section{FUNDING}

This work was supported by the Canadian Institutes of Health Research (CIHR) (grants PJT-156416 and MOP-125990 to AD), by the Conselho Nacional de Pesquisa e Desenvolvimento (CNPq) (grant 305065/2016-5 to RS), and the Fundação de Amparo do Estado de Minas Gerais (FAPEMIG) (grant 0020218 to $\mathrm{RS}$ ). AD was the holder of the Canada Research Chair on the Biology of intracellular parasitism. TS was the recipient of a scholarship from FAPEMIG and from the Emerging Leaders in the Americas Program (Global Affairs Canada). GA was supported by a CIHR Banting and Best Doctoral Award.

\section{ACKNOWLEDGMENTS}

We thank W. R. McMaster (University of British Columbia) for providing antibodies against GP63, A. Jardim (McGill University) for providing the aldolase antiserum, and J. Tremblay for assistance with confocal immunofluorescence experiments.

Impact on innate immune response and macrophage functions. Cell. Immunol. 309, 7-18. doi: 10.1016/j.cellimm.2016.07.013

Bussotti, G., Gouzelou, E., Côrtes Boité M., Kherachi, I., Harrat, Z., Eddaikra, N., et al. (2018). Leishmania genome dynamics during environmental adaptation reveal strain-specific differences in gene copy number variation, karyotype instability, and telomeric amplification. mBio 9:e01399-18. doi: 10.1128/mBio.01399-18

Button, L. L., Wilson, G., Astell, C. R., and McMaster, W. R. (1993). Recombinant Leishmania surface glycoprotein GP63 is secreted in the baculovirus expression system as a latent metalloproteinase. Gene 134, 75-81. doi: 10.1016/0378-1119(93)90176-4

Canton, J., and Kima, P. E. (2012). Targeting host syntaxin-5 preferentially blocks Leishmania parasitophorous vacuole development in infected cells and limits experimental leishmania infections. Am. J. Pathol. 181, 1348-1355. doi: 10.1016/j.ajpath.2012.06.041

Casgrain, P. A., Martel, C., McMaster, W. R., Mottram, J. C., Olivier, M., and Descoteaux, A. (2016). Cysteine peptidase B regulates Leishmania mexicana virulence through the modulation of GP63 expression. PLoS Pathog. 12:e1005658. doi: 10.1371/journal.ppat.1005658

Cebrian, I., Visentin, G., Blanchard, N., Jouve, M., Bobard, A., Moita, C., et al. (2011). Sec22b regulates phagosomal maturation and antigen crosspresentation by dendritic cells. Cell 147, 1355-1368. doi: 10.1016/j.cell.2011.11.021

Chaudhuri, G., Chaudhuri, M., Pan, A., and Chang, K. P. (1989). Surface acid proteinase (gp63) of Leishmania mexicana. A metalloenzyme capable of protecting liposome-encapsulated proteins from phagolysosomal degradation by macrophages. J. Biol. Chem. 264, 7483-7489. 
de Carvalho, R. V. H., Andrade, W. A., Lima-Junior, D. S., Dilucca, M., de Oliveira, C. V., Wang, K., et al. (2019). Leishmania lipophosphoglycan triggers caspase11 and the non-canonical activation of the NLRP3 inflammasome. Cell Rep. 26:e5. doi: 10.1016/j.celrep.2018.12.047

de Chaumont, F., Dallongeville, S., Chenouard, N., Hervé N., Pop, S., Provoost, T., et al. (2012). Icy: an open bioimage informatics platform for extended reproducible research. Nat. Methods 9:690. doi: 10.1038/nmeth.2075

Descoteaux, A., and Matlashewski, G. (1989). c-fos and tumor necrosis factor gene expression in Leishmania donovani-infected macrophages. Mol. Cell Biol. 9, 5223-5227. doi: 10.1128/MCB.9.11.5223

Descoteaux, A., Turco, S. J., Sacks, D. L., and Matlashewski, G. (1991). Leishmania donovani lipophosphoglycan selectively inhibits signal transduction in macrophages. J. Immunol. 146, 2747-2753.

Desjardins, M., and Descoteaux, A. (1997). Inhibition of phagolysosomal biogenesis by the Leishmania lipophosphoglycan. J. Exp. Med. 185, 2061-2068. doi: $10.1084 /$ jem.185.12.2061

Guimarães, L. H., Machado, P. R., Lago, E. L., Morgan, D. J., Schriefer, A., Bacellar, O., et al. (2009). Atypical manifestations of tegumentary leishmaniasis in a transmission area of Leishmania braziliensis in the state of Bahia, Brazil. Trans R Soc. Trop. Med. Hyg. 103, 712-715. doi: 10.1016/j.trstmh.2009.04.019

Hassani, K., Shio, M. T., Martel, C., Faubert, D., and Olivier, M. (2014). Absence of metalloprotease GP63 alters the protein content of Leishmania exosomes. PLoS ONE 9:e95007. doi: 10.1371/journal.pone.0095007

Heinzel, F. P., Sadick, M. D., Holaday, B. J., Coffman, R. L., and Locksley, R. M. (1989). Reciprocal expression of interferon gamma or interleukin 4 during the resolution or progression of murine leishmaniasis. Evidence for expansion of distinct helper T cell subsets. J. Exp. Med. 169, 59-72. doi: 10.1084/jem.169.1.59

Huynh, K. K., Eskelinen, E. L., Scott, C. C., Malevanets, A., Saftig, P., and Grinstein, S. (2007). LAMP proteins are required for fusion of lysosomes with phagosomes. EMBO J. 26, 313-24. doi: 10.1038/sj.emboj.7601511

Ilg, T. (2000). Lipophosphoglycan is not required for infection of macrophages or mice by Leishmania mexicana. EMBO J. 19, 1953-1962. doi: $10.1093 / \mathrm{emboj} / 19.9 .1953$

Ilg, T., Demar, M., and Harbecke, D. (2001). Phosphoglycan repeat-deficient Leishmania mexicana parasites remain infectious to macrophages and mice. J. Biol. Chem. 276, 4988-4997. doi: 10.1074/jbc.M008030200

Joshi, P. B., Kelly, B. L., Kamhawi, S., Sacks, D. L., and McMaster, W. R. (2002). Targeted gene deletion in Leishmania major identifies leishmanolysin (GP63) as a virulence factor. Mol. Biochem. Parasitol. 120, 33-40. doi: 10.1016/S0166-6851(01)00432-7

Lázaro-Souza, M., Matte, C., Lima, J. B., Arango Duque, G., QuintelaCarvalho, G., de Carvalho Vivarini, Á., et al. (2018). Leishmania infantum lipophosphoglycan-deficient mutants: a tool to study host cell-parasite interplay. Front. Microbiol. 9:626. doi: 10.3389/fmicb.2018.00626

Lerm, M., Holm, A., Seiron, A., Särndahl, E., Magnusson, K. E., and Rasmusson, B. (2006). Leishmania donovani requires functional Cdc42 and Rac1 to prevent phagosomal maturation. Infect Immun. 74, 2613-2618. doi: 10.1128/IAI.74.5.2613-2618.2006

Lodge, R., Diallo, T. O., and Descoteaux, A. (2006). Leishmania donovani lipophosphoglycan blocks NADPH oxidase assembly at the phagosome membrane. Cell Microbiol. 8, 1922-1931. doi: $10.1111 /$ j.1462-5822.2006.00758.x

Macdonald, M. H., Morrison, C. J., and McMaster, W. R. (1995). Analysis of the active site and activation mechanism of the Leishmania surface metalloproteinase GP63. Biochim. Biophys. 1253, 199-207. doi: 10.1016/0167-4838(95)00155-5

Martínez-López, M., Soto, M., Iborra, S., and Sancho, D. (2018). Leishmania hijacks myeloid cells for immune escape. Front. Microbiol. 9:883. doi: $10.3389 /$ fmicb.2018.00883

Matheoud, D., Moradin, N., Bellemare-Pelletier, A., Shio, M. T., Hong, W. J., Olivier, M., et al. (2013). Leishmania evades host immunity by inhibiting antigen cross-presentation through direct cleavage of the SNARE VAMP8. Cell Host Microbe 14, 15-25. doi: 10.1016/j.chom.2013. 06.003

Matte, C., Casgrain, P.-A., Séguin, O., Moradin, N., Hong, W. J., and Descoteaux, A. (2016). Leishmania major promastigotes evade LC3-associated phagocytosis through the action of GP63. PLoS Pathog. 12:e1005690. doi: 10.1371/journal.ppat.1005690
Matte, C., and Descoteaux, A. (2016). Exploitation of the host cell membrane fusion machinery by leishmania is part of the infection process. PLoS Pathog. 12:e1005962. doi: 10.1371/journal.ppat.1005962

Medina, L. S., Souza, B. A., Queiroz, A., Guimarães, L. H., Lima Machado, P. R., Carvalho, M., et al. (2016). The gp63 gene cluster is highly polymorphic in natural Leishmania (Viannia) braziliensis populations, but functional sites are conserved. PLoS ONE 11:e0163284. doi: 10.1371/journal.pone.0163284

Moradin, N., and Descoteaux, A. (2012). Leishmania promastigotes: building a safe niche within macrophages. Front Cell Infect. Microbiol. 2:121. doi: $10.3389 /$ fcimb.2012.00121

Noben-Trauth, N., Kropf, P., and Müller, I. (1996). Susceptibility to Leishmania major infection in interleukin-4-deficient mice. Science 271, 987-990. doi: $10.1126 /$ science.271.5251.987

Noben-Trauth, N., Paul, W. E., and Sacks, D. L. (1999). IL-4- and IL-4 receptordeficient $\mathrm{BALB} / \mathrm{c}$ mice reveal differences in susceptibility to Leishmania major parasite substrains. J. Immunol. 162, 6132-6140.

Olivier, M., Atayde, V. D., Isnard, A., Hassani, K., and Shio, M. T. (2012). Leishmania virulence factors: focus on the metalloprotease GP63. Microbes Infect. 14, 1377-1389. doi: 10.1016/j.micinf.2012.05.014

PAHO/WH1O (2017). Leishmaniasis: Epidemiological Report of the Americas Washington: Pan American Health Organization [cited 2019 February 25]. 5. Available online at: http://www.paho.org/leishmaniasis

Podinovskaia, M., and Descoteaux, A. (2015). Leishmania and the macrophage: a multifaceted interaction. Fut. Microbiol. 10, 111-129. doi: 10.2217/fmb.14.103

Quaresma, P. F., de Brito, C. F. A., Rugani, J. M. N., Freire Jd, M., Baptista Rd, P., Moreno, E. C., et al. (2018). Distinct genetic profiles of Leishmania (Viannia) braziliensis associate with clinical variations in cutaneous-leishmaniasis patients from an endemic area in Brazil. Parasitology 145, 1161-1169. doi: $10.1017 /$ S0031182018000276

Rueden, C. T., Schindelin, J., Hiner, M. C., DeZonia, B. E., Walter, A. E., Arena, E. T., et al. (2017). ImageJ2: imageJ for the next generation of scientific image data. BMC Bioinform. 18:529. doi: 10.1186/s12859-017-1934-z

Rugani, J. N., Quaresma, P. F., Gontijo, C. F., Soares, R. P., and MonteNeto, R. L. (2018). Intraspecies susceptibility of Leishmania (Viannia) braziliensis to antileishmanial drugs: antimony resistance in human isolates from atypical lesions. Biomed. Pharmacother. 108, 1170-1180. doi: 10.1016/j.biopha.2018.09.149

Scianimanico, S., Desrosiers, M., Dermine, J. F., Méresse, S., Descoteaux, A., and Desjardins, M. (1999). Impaired recruitment of the small GTPase rab7 correlates with the inhibition of phagosome maturation by Leishmania donovani promastigotes. Cell Microbiol. 1, 19-32. doi: 10.1046/j.1462-5822.1999.00002.x

Séguin, O., and Descoteaux, A. (2016). Leishmania, the phagosome, and host responses: the journey of a parasite. Cell. Immunol. 309, 1-6. doi: 10.1016/j.cellimm.2016.08.004

Shio, M. T., Hassani, K., Isnard, A., Ralph, B., Contreras, I., Gómez, M. A., et al. (2012). Host cell signalling and Leishmania mechanisms of evasion. J. Trop. Med. 2012:819512. doi: 10.1155/2012/819512

Soares, R. P., Cardoso, T. L., Barron, T., Araújo, M. S. S., Pimenta, P. F., and Turco, S. J. (2005). Leishmania braziliensis: a novel mechanism in the lipophosphoglycan regulation during metacyclogenesis. Int. J. Parasitol. 35, 245-253. doi: 10.1016/j.ijpara.2004.12.008

Soares, R. P., Macedo, M. E., Ropert, C., Gontijo, N. F., Almeida, I. C., Gazzinelli, R. T., et al. (2002). Leishmania chagasi: lipophosphoglycan characterization and binding to the midgut of the sand fly vector Lutzomyia longipalpis. Mol. Biochem. Parasitol. 121, 213-224. doi: 10.1016/S0166-6851(02)00033-6

Späth, G. F., Epstein, L., Leader, B., Singer, S. M., Avila, H. A., Turco, S. J., et al. (2000). Lipophosphoglycan is a virulence factor distinct from related glycoconjugates in the protozoan parasite Leishmania major. Proc. Natl. Acad. Sci. U.S.A. 97, 9258-9263. doi: 10.1073/pnas. 160257897

Späth, G. F., Garraway, L. A., Turco, S. J., and Beverley, S. M. (2003). The role(s) of lipophosphoglycan (LPG) in the establishment of Leishmania major infections in mammalian hosts. Proc. Natl. Acad. Sci. U.S.A. 100, 9536-9541. doi: 10.1073/pnas.1530604100

Sutter, A., Antunes, D., Silva-Almeida, M., Costa MGd, S., and Caffarena, E. R. (2017). Structural insights into leishmanolysins encoded on chromosome 10 of Leishmania (Viannia) braziliensis. Memórias do Instituto Oswaldo Cruz 112, 617-625. doi: 10.1590/0074-02760160522 
Tolson, D. L., Turco, S. J., Beecroft, R. P., and Pearson, T. W. (1989). The immunochemical structure and surface arrangement of Leishmania donovani lipophosphoglycan determined using monoclonal antibodies. Mol. Biochem. Parasitol. 35, 109-118. doi: 10.1016/0166-6851(89)90113-8

Verma, J. K., Rastogi, R., and Mukhopadhyay, A. (2017). Leishmania donovani resides in modified early endosomes by upregulating Rab5a expression via the downregulation of miR-494. PLoS Pathog. 13:e1006459. doi: 10.1371/journal.ppat.1006459

Vieira Td, S., Rugani, J. N., Nogueira, P. M., Torrecilhas, A. C., Gontijo, C. M. F., Descoteaux, A., et al. (2019). Intraspecies polymorphisms in the lipophosphoglycan of L. braziliensis differentially modulate macrophage activation via TLR4. Front. Cell. Infect. Microbiol. 9:240. doi: 10.3389/fcimb.2019.00240

Vinet, A. F., Fukuda, M., and Descoteaux, A. (2008). The exocytosis regulator synaptotagmin $\mathrm{V}$ controls phagocytosis in macrophages. J. Immunol. 181, 5289-5295.doi: 10.4049/jimmunol.181.8.5289
Vinet, A. F., Fukuda, M., Turco, S. J., and Descoteaux, A. (2009). The Leishmania donovani lipophosphoglycan excludes the vesicular proton-ATPase from phagosomes by impairing the recruitment of synaptotagmin V. PLoS Pathog. 5:e1000628. doi: 10.1371/journal.ppat.1000628

Conflict of Interest Statement: The authors declare that the research was conducted in the absence of any commercial or financial relationships that could be construed as a potential conflict of interest.

Copyright (c) 2019 da Silva Vieira, Arango Duque, Ory, Gontijo, Soares and Descoteaux. This is an open-access article distributed under the terms of the Creative Commons Attribution License (CC BY). The use, distribution or reproduction in other forums is permitted, provided the original author(s) and the copyright owner(s) are credited and that the original publication in this journal is cited, in accordance with accepted academic practice. No use, distribution or reproduction is permitted which does not comply with these terms. 